\title{
FATORES RELACIONADOS À QUALIDADE DE VIDA DA MULHER IDOSA NO MUNICIPIO DE GUANAMBI (BA)
}

\author{
Núbia Rêgo Santos ${ }^{1}$ \\ Cinoélia Leal de Souza² \\ Simone Aline Ferreira ${ }^{3}$ \\ Jaqueline Pereira Alves ${ }^{4}$ \\ Victor Neves Reis ${ }^{5}$ \\ Elaine Santos da Silva ${ }^{6}$
}

resumo

A qualidade de vida é um conjunto de fatores subjetivos e objetivos, que deve ser avaliada, sobretudo no envelhecimento, para compreender as necessidades de vida e saúde dos idosos e propor estratégias de melhoria da qualidade de vida de cada indivíduo. Objetivo: analisar os fatores relacionados à qualidade de vida da

1 Graduada em Enfermagem pelo Centro Universitário UNIFG. E-mail: nubya.net12@gmail.com.

2 Graduada em Enfermagem. Doutora em Ciências da Saúde. Professora no Centro Universitário UNIFG, vinculada ao Colegiado de Enfermagem. E-mail: cinoelia5@hotmail.com.

3 Graduada em Enfermagem pelo Centro Universitário UNIFG. E-mail: simonealine32@gmail.com.

4 Graduada em Enfermagem pelo Centro Universitário UNIFG. E-mail: jaqueline151983@ hotmail.com.

5 Graduado em Enfermagem pelo Centro Universitário UNIFG. E-mail: victor_gbi14@yahoo.com.

6 Graduada em Enfermagem pelo Centro Universitário UNIFG. E-mail: elaine-ss@live.com. 
mulher idosa na cidade de Guanambi (BA), no ano de 2016. Materiais e Métodos: estudo quantitativo, transversal e de caráter descritivo, realizado com 550 idosas. Os instrumentos utilizados na coleta de dados foram um formulário semi-estruturado de diagnóstico situacional e o World Health Organization Quality of Life (WHOQOL-Bref) para mensurar a qualidade de vida desse segmento populacional. A análise foi realizada por meio da estatística univariada, com cálculos de Qui-Quadrado $\chi^{2}$, e regressão logística simples. Resultados: As idosas eram em sua maioria analfabetas, aposentadas, sem cônjuge, negras, vivendo em lares multigeracionais e 68,2\% com uma percepção positiva quanto a sua qualidade de vida, mesmo vivendo em situações adversas. Os fatores que estiveram relacionados à qualidade de vida foram os sociodemográficos, condições de saúde e o estilo de vida, sendo que esses fatores apresentados negativamente contribuem para maior incapacidade, vulnerabilidade e dependência da idosa. Conclusão: O bem-estar da idosa é afetado pela redução de recursos nas dimensões educacionais, psicossociais e econômicas, com isso, a pesquisa sinalizou a necessidade de implantação de ações, voltadas para assistência de saúde, atividades recreativas e de socialização, que possibilitem autonomia e independência, para a melhoria da qualidade de vida.

palavras-chave

Fatores de Risco. Envelhecimento Populacional. Saúde da Mulher.

\section{Introdução}

Envelhecer é um processo natural, no qual a pessoa tende a ficar gradativamente mais vulnerável e dependente para exercer as suas atividades de vida diárias. Por critério de definição, o Estatuto do Idoso brasileiro considera idosa a pessoa com idade igual ou superior 60 anos, e traz o envelhecimento como um direito personalíssimo e social (CARTAXO et al., 2012; KÜCHEMANN, 2012).

$\mathrm{O}$ aumento do número de idosos tem ocorrido de maneira global, decorrente de alterações demográficas nas últimas décadas, estando constantemente associado às transformações do perfil epidemiológico e às características econômicas e sociais das populações, pois o envelhecimento envolve interligações multidisciplinares, caracterizando-se como um processo complexo (CAMPOS; FERREIRA; VARGAS, 2015; OLIVEIRA; NOVAES, 2013). A população idosa do 
Brasil também vem aumentando consideravelmente. Segundo dados do Instituto Brasileiro de Geografia e Estatística - IBGE (2015), as taxas de crescimento desse segmento populacional serão de mais de $4 \%$ ao ano no período de 2012 a 2022. O percentual de pessoas idosas passa de 14,2 milhões, podendo atingir 73,5 milhões em 2060. Correspondendo a 12,6\% da população brasileira total, podendo atingir a $20 \%$ da população em 2050 . Desse universo, o sexo feminino representava 55,7\% comparado ao sexo masculino (CASTRO; BARROS JÚNIOR, 2016; STEDILE; MARTINI; SCHMIDT, 2017; CAMPOS; GONÇALVES, 2018). A razão para este fato é a expectativa de vida maior para as mulheres, que vivem em média 8 anos a mais que os homens (KÜCHEMANN, 2012).

Dentre os fatores que promovem uma maior expectativa de vida nas mulheres está o menor consumo do tabaco e álcool e à maior demanda por assistência à saúde. Tem-se também uma maior exposição dos homens jovens e adultos às mortes violentas por causas externas, como acidentes e homicídios (CHAIMOWICZ, 2013). Há ainda um maior número de mulheres entre os idosos, principalmente acima de 80 anos (KÜCHEMANN, 2012). Essa predominância de mulheres na população de idosos tem sido caracterizada inclusive como uma "feminilização da velhice" (STEDILE; MARTINI; SCHMIDT, 2017).

Nesse sentido, é necessário que o idoso se mantenha ativo e envolvido com as atividades diárias, através da realização de exercícios físicos, sociais e intelectuais, conservando sua autonomia e independência. Desse modo, é importante que o sentido de envelhecer oportunize ao indivíduo a escolha por hábitos de vida saudáveis, proporcionando o controle da saúde e condicionando uma melhora na qualidade de vida, decorrente do bem-estar emocional, que envolve também o relacionamento social (FEHLBERG, 2013; CAMPOS; FERREIRA; VARGAS, 2015).

A qualidade de vida é um reflexo das relações e sentimentos subjetivos, como o amor, inserção e realização social, liberdade e dos fatores objetivos, como alimentação, lazer, educação, saúde e habitação, que proporciona conforto, realização individual e coletiva (VIEIRA et al., 2012). O conceito de qualidade de vida "envolve a percepção do indivíduo de sua posição na vida em relação ao contexto e sistemas de valores nos quais se insere bem como seus objetivos, padrões, expectativas e preocupações" (WHO, 1998). Nota-se que esse é um conceito complexo, compreendendo a saúde física, vínculo sociais, estado psíquico, nível de independência e a interação do indivíduo com o ambiente que o cerca.

A qualidade de vida está diretamente associada também à disponibilidade de recursos sociais utilizados e vivenciados no percurso da vida e no próprio processo do envelhecimento. Assim sendo, a carência ou excesso desses riscos 
socioeconômicos tem interferência na velhice, como por exemplo: a renda, o grau de escolaridade e condições de saneamento básico entre outros (CARTAXO et al., 2012; VIEIRA et al., 2012).

Nessa perspectiva, salienta-se a importância científica e social de se analisar as condições que afetam a senescência e os aspectos ligados à qualidade de vida de idosos, sobretudo da mulher, com a finalidade de desenvolver alternativas de intervenção e sugerir ações e políticas públicas no âmbito da saúde, buscando satisfazer às demandas da população nessa faixa etária (DAWALIBI; GOULART; PREARO, 2014). Diante disso, o presente estudo objetivou analisar os fatores relacionados à qualidade de vida da mulher idosa, no município de Guanambi (BA), no ano de 2016.

\section{Materiais e métodos}

\subsection{Tipo de estudo}

Tratou-se de um estudo quantitativo, transversal e de caráter descritivo, pois a pesquisa quantitativa leva em consideração que tudo pode ser mensurável em números, opiniões e informações para agrupá-los e analisá-los (PRONDANOW, 2013). A pesquisa transversal busca analisar os dados em um determinado período de tempo, para uma amostra ou população definida. Já as pesquisas descritivas objetivam relatar as características de determinada população ou fenômeno ou o estabelecer relações entre variáveis (GIL, 2010).

\subsection{Local do estudo}

A pesquisa foi realizada no município de Guanambi, estado da Bahia, situado a $796 \mathrm{~km}$ de sua capital (Salvador), que possui uma área territorial de 1.301,80 km2. A estimativa da população em 2013 para este município constatou 84.645 habitantes, dos quais o idoso representa cerca de $10,8 \%$ ou seja desse universo, as idosas representando aproximadamente de 6,5\% da população total.

\subsection{População e amostra}

O cálculo da amostra ocorreu através de amostragem probabilística simples, sem reposição. Para o cálculo amostral foi considerado o número total 
de mulheres do município estudado ( $\mathrm{N}=40$ 352), segundo estimativa do IBGE (2010), adotando a frequência esperada de mulheres idosas de $12 \%$, e $3 \%$ por diferença estatisticamente significativa de $95 \%$, o que resultou em 485 idosas. Considerando as perdas, acrescentou-se $20 \%$, que resultou em 582 mulheres, a partir da amostra, incluindo a perda de 32 participantes por desistência e óbito, no final do estudo foram incluídas 550 idosas. A amostra final foi de 550 idosas do município de Guanambi (BA). Os critérios de elegibilidade do estudo foram: idosas com idade maior ou igual a 60 anos, que estivessem conscientes e aceitassem participar da pesquisa, mediante assinatura do Termo de Consentimento Livre e Esclarecido (TCLE).

\subsection{Coleta de dados}

A coleta de dados ocorreu de janeiro de 2016 a abril de 2016 em quatorze bairros do município. A amostra probabilística simples em que todos os elementos da amostra têm a chance de escolha, e com base no número total das idosas (PRONDANOW, 2013).

Para efetuar a coleta, foi realizada uma abordagem nas residências das participantes, após mapeamento obtido mediante cadastro nas Unidades de Saúde da Família (USF) dos respectivos bairros. A princípio, foi realizado o levantamento aleatório da localização das idosas, juntamente com a os profissionais das unidades. para sucessivamente, a mesma ser entrevistada pela equipe de coleta composta por nove coletadores, discentes do terceiro ao oitavo semestre do curso de graduação em enfermagem, antecipadamente treinados na aplicação dos instrumentos de coleta de dados. As idosas em sua grande maioria aceitaram participar da pesquisa. Nesse caso, foi agendado o dia e horário para a aplicação dos questionários em seus domicílios, com duração das entrevistas entre 40 e 50 minutos.

\subsection{Instrumentos para coleta de dados}

Os instrumentos utilizados na pesquisa foram um formulário semiestruturado que buscou contemplar as informações dos aspectos de saúde e sociodemográficas das idosas, e o instrumento de avaliação da Qualidade de Vida da Organização Mundial da Saúde (OMS), conhecido como o World Health Organization Quality of Life (WHOQOL). Na versão abreviada em português, o WHOQOL-Bref é composto por 26 perguntas, sendo duas gerais sobre qualidade de vida geral e satisfação com a saúde e as demais 24 caracterizando 
cada uma das 24 facetas que constitui o instrumento original. É constituído de quatros domínios: físico, psicológico, relações sociais e meio ambiente, sendo que, quanto mais alto o escore melhor a qualidade de vida, no entanto, não há ponto de corte para sua classificação (ALVES et al., 2009; VITORINO; PASKULIN; VIANNA, 2013).

\subsection{Análise e tratamento dos dados}

Para análise da percepção da qualidade de vida foi utilizado os escores adequado e inadequado, conforme estabelecido pelo próprio instrumento de coleta de dados. Foi feita uma análise estatística univariada, com cálculos de Qui-Quadrado $\chi 2$, para avaliar as variáveis sociodemográficas citadas anteriormente e sua relação com a percepção da qualidade de vida. Realizou-se também uma análise de regressão logística para identificar os principais fatores associados à qualidade de vida das idosas, na qual a variável dependente foi a percepção da qualidade de vida e as variáveis independentes foram as que se mostraram significativas na análise univariada, assim como as variáveis que não foram significativas. Os dados foram tabulados utilizando o programa de computador Microsoft Excel ${ }^{\circledR}$, analisado por meio do programa estatístico Stata versão 10 .

\subsection{Questões éticas}

Todas as fases deste projeto de pesquisa foram realizadas em consonância com as questões ético-legais da resolução n. 466/12 do Conselho Nacional de Saúde que regulamenta as pesquisas envolvendo seres humanos no Brasil. O presente projeto foi aprovado pelo Comitê de Ética e Pesquisa da Faculdade Independente do Nordeste (FAINOR), sob o protocolo CAAE: 50695415.5.0000.5578.

\section{Resultados}

Das 550 idosas participantes da pesquisa, a maioria possuía idade entre 60 a 70 anos (54,1\%), sem companheiro (53,8\%) e se autodeclararam da raça/cor da pele negra, que foi a junção de pardas e pretas (54,4\%). Quanto ao nível de escolaridade, a maioria $(53,8 \%)$ das idosas cursou apenas o ensino fundamental e 39,5\% eram analfabetas. No que se refere à renda familiar, $60,4 \%$ recebem um salário mínimo por mês (Tabela 1). 
Tabela 1 - Características sociodemográficas das idosas residentes no município de Guanambi (BA) no ano de 2016.

\begin{tabular}{lll}
\hline Variáveis & N & $\%$ \\
\hline Idade & 297 & 54,1 \\
60 a 70 anos & 206 & 37,5 \\
71 a 80 anos & 46 & 8,4 \\
81 ou mais anos & & \\
Situação conjugal & 254 & 46,2 \\
Com companheiro & 296 & 53,8 \\
Sem companheiro & & \\
Escolaridade & 217 & 39,5 \\
Analfabeta & 296 & 53,8 \\
Até 8 anos de estudos & 37 & 6,7 \\
Mais de 8 anos de estudos & & \\
Raça/cor da pele & 251 & 45,6 \\
Não negra & 299 & 54,4 \\
Negra & & \\
Ocupação remunerada & 481 & 87,6 \\
Não & 68 & 12,4 \\
Sim & & 36,1 \\
Renda & 330 & 60,4 \\
Até 1 salário & 199 & 36,4 \\
Ma 3 salários & & \\
Mais de 3 salários & & \\
\hline
\end{tabular}

Fonte: Tabela elaborada pelos autores.

Legenda: $\mathrm{N}$ = número; \% = porcentagem.

Quanto à cooperação familiar na renda da família, $71,8 \%$ das idosas não tinham ajuda financeira, sendo sua renda a única para sustentar toda a família. As idosas moravam, em sua maioria, com mais de três familiares (32,4\%). Quanto ao tipo de moradia, $95,1 \%$ das idosas possuem casa própria, com mais de seis cômodos (70,7\%) (Tabela 2). 
Tabela 2 - Caracterização das condições de vida das idosas residentes no município de Guanambi (BA) no ano de 2016.

\begin{tabular}{llc}
\hline Variáveis & N & $\%$ \\
\hline Habitação & 523 & 95,1 \\
Própria & 18 & 3,3 \\
Alugada & 08 & 1,5 \\
Cedida & 01 & 0,2 \\
Outra & & \\
Número de cômodos & 55 & 10,0 \\
Até 4 & 495 & 90,0 \\
5 a 6 & & \\
Número de pessoas & 55 & 10,0 \\
1 & 149 & 27,1 \\
2 & 168 & 30,5 \\
3 & 178 & 32,4 \\
Mais de 3 & & \\
Cooperação familiar na renda & 155 & 28,2 \\
Sim & 395 & 71,8 \\
Não
\end{tabular}

Fonte: Tabela elaborada pelos autores.

Legenda: $\mathrm{N}$ = número; \% = porcentagem.

Os resultados mostraram que 68,2\% das idosas têm uma percepção positiva quanto a sua qualidade de vida. A análise estatística univariada (Tabela 3), apontou apenas a renda (p 0,002) como variável estatisticamente associada à percepção da qualidade de vida. Observou-se que 69,8\% dos sujeitos com renda familiar com mais de três salários mínimos apresentaram percepção de qualidade de vida negativa. 
Tabela 3 - Características sociodemográficas segundo a percepção da qualidade de vida das idosas residentes no município de Guanambi (BA) no ano de 2016.

\begin{tabular}{|c|c|c|c|c|c|}
\hline \multirow{3}{*}{ Variáveis } & \multicolumn{4}{|c|}{ Percepção da qualidade de vida } & \multirow{3}{*}{$\mathrm{p}$-valor } \\
\hline & \multicolumn{2}{|c|}{ Inadequada } & \multicolumn{2}{|c|}{ Adequada } & \\
\hline & $\mathrm{N}$ & $\%$ & $\mathrm{~N}$ & $\%$ & \\
\hline \multicolumn{6}{|l|}{ Idade } \\
\hline 60 a 70 anos & 97 & 32,7 & 200 & 67,3 & \multirow{3}{*}{0,671} \\
\hline 71 a 80 anos & 66 & 32,0 & 140 & 68,0 & \\
\hline 81 ou mais anos & 12 & 26,1 & 34 & 73,9 & \\
\hline \multicolumn{6}{|l|}{ Situação conjugal } \\
\hline Com companheiro & 78 & 30,7 & 176 & 69,3 & \multirow{2}{*}{0,605} \\
\hline Sem companheiro & 97 & 32,8 & 199 & 67,2 & \\
\hline \multicolumn{6}{|l|}{ Raça/cor da pele } \\
\hline Não negra & 78 & 44,6 & 173 & 46,1 & \multirow{2}{*}{0,732} \\
\hline Negra & 97 & 55,4 & 202 & 53,9 & \\
\hline \multicolumn{6}{|c|}{ Ocupação remunerada } \\
\hline Não & 155 & 89,1 & 326 & 86,9 & \multirow{2}{*}{0,477} \\
\hline Sim & 19 & 10,9 & 49 & 13,1 & \\
\hline \multicolumn{6}{|l|}{ Renda } \\
\hline Até 1 salário & 01 & 0,6 & 16 & 4,3 & \multirow{3}{*}{0,002} \\
\hline 2 a 3 salários & 51 & 29,7 & 148 & 39,7 & \\
\hline Mais de 3 salários & 120 & 69,8 & 209 & 56,0 & \\
\hline
\end{tabular}

Fonte: Tabela elaborada pelos autores.

Legenda: $\mathrm{N}$ = número; \% = porcentagem.

Na análise das condições de vida, cinco variáveis apresentaram significância estatística relacionada com a percepção da qualidade de vida: percepção da saúde, recursos financeiros, lazer, condições de moradia e transporte (Tabela 4). Essas variáveis identificadas como significativas na análise foram utilizadas no modelo multivariado de regressão logística, juntamente com as variáveis que não foram significativas, coletadas no questionário sociodemográfico. 
Tabela 4 - Avaliação das condições de vida segundo a percepção da qualidade de vida das idosas residentes no município de Guanambi (BA) no ano de 2016.

\begin{tabular}{|c|c|c|c|c|c|}
\hline \multirow{3}{*}{ Variáveis } & \multicolumn{4}{|c|}{ Percepção da qualidade de vida } & \multirow{3}{*}{$\mathrm{p}$-valor } \\
\hline & \multicolumn{2}{|c|}{ Inadequada } & \multicolumn{2}{|c|}{ Adequada } & \\
\hline & $\mathrm{N}$ & $\%$ & $\mathrm{~N}$ & $\%$ & \\
\hline \multicolumn{6}{|c|}{ Percepção da saúde } \\
\hline Adequada & 75 & 43,6 & 289 & 77,5 & \multirow{2}{*}{0,000} \\
\hline Inadequada & 97 & 56,4 & 84 & 22,5 & \\
\hline \multicolumn{6}{|c|}{ Recurso financeiro } \\
\hline Suficiente & 35 & 20,4 & 133 & 35,7 & \multirow{2}{*}{0,000} \\
\hline Insuficiente & 137 & 79,7 & 240 & 64,3 & \\
\hline \multicolumn{6}{|l|}{ Lazer } \\
\hline Suficiente & 30 & 17,4 & 141 & 37,8 & \multirow{2}{*}{0,000} \\
\hline Insuficiente & 142 & 82,6 & 232 & 62,2 & \\
\hline \multicolumn{6}{|c|}{ Condições de moradia } \\
\hline Adequadas & 132 & 76,7 & 346 & 92,8 & \multirow{2}{*}{0,000} \\
\hline Inadequadas & 40 & 23,3 & 27 & 7,2 & \\
\hline \multicolumn{6}{|l|}{ Transporte } \\
\hline Adequado & 75 & 43,6 & 206 & 55,2 & \multirow{2}{*}{0,012} \\
\hline Inadequado & 97 & 56,4 & 167 & 44,8 & \\
\hline \multicolumn{6}{|c|}{ Sentimentos negativos } \\
\hline Não & 06 & 3,5 & 11 & 3,0 & \multirow{2}{*}{0,736} \\
\hline Sim & 166 & 96,5 & 362 & 97,0 & \\
\hline
\end{tabular}

Fonte: Tabela elaborada pelos autores.

Legenda: $\mathrm{N}=$ número; \% = porcentagem.

Na análise estatística multivariada de regressão logística notou-se que as variáveis que se mantiveram no modelo final, como fatores associados à qualidade de vida das idosas, foram: a renda ( $\mathrm{OR}=1,62$; IC =1,16 - 2,26), a percepção da saúde $(\mathrm{OR}=4,16 ; \mathrm{IC}=2,77-6,25)$, lazer $(\mathrm{OR}=2,03 ; \mathrm{IC}=1,25-3,27)$ e a condição de moradia $(\mathrm{OR}=3,10 ; \mathrm{IC}=1,74-5,53)$. As demais variáveis não se mantiveram no modelo multivariado (Tabela 5). 
Tabela 5 - Resultados da análise de regressão logística para os fatores associados à percepção da qualidade de vida das idosas residentes do município de Guanambi (BA) no ano de 2016.

\begin{tabular}{llll}
\hline Variáveis & OR & IC (95\%) & p-valor \\
\hline Renda familiar & 1,62 & $1,16-2,26$ & 0,005 \\
Percepção da saúde & 4,16 & $2,77-6,25$ & 0,000 \\
Lazer & 2,03 & $1,25-3,27$ & 0,004 \\
Condições de moradia & 3,10 & $1,74-5,53$ & 0,000 \\
\hline
\end{tabular}

Fonte: Tabela elaborada pelos autores.

Legenda: OR: Odds ratio; IC: Intervalo de confiança.

\section{Discussão}

O envelhecimento saudável depende da relação multidimensional, tais como, idade, sexo, escolaridade, estado civil, lazer, contexto socioeconômico e percepção de saúde, que podem influenciar de forma direta ou indireta no bem-estar e na percepção do idoso sobre a sua qualidade de vida (VISENTIN et al., 2015; VAGETTI et al., 2013). É notado nos diversos aspectos, como menores níveis de renda, aumento da idade, limitações físicas e o sexo feminino estão associados à presença de sintomas de depressão, bem como, menores níveis de alfabetização e a inferioridade na autopercepção do estado de saúde (ALBERTE; RUSCALLEDA; GUARIENTO, 2015). Em estudo realizado em Jequié (BA), mencionado por Santos e Cunha (2014), 70\% das idosas apresentaram baixo nível socioeconômico, se enquadrando nas classes econômicas " $\mathrm{D}$ e E". Sendo que $60,6 \%$ dessas idosas eram aposentadas, e apontavam a aposentadoria como a principal fonte de sobrevivência, além do mais, 13,9\% estavam inseridas no mercado de trabalho, como diarista, para suprir as necessidades básicas.

Percebe-se que as condições socioeconômicas estiveram fortemente associadas ao envelhecimento (CAMPOS; GONÇALVES, 2018). Constata-se ainda que, a qualidade de vida está associada ao contexto socioeconômico das pessoas, por isso, as condições socioeconômicas desfavoráveis para as idosas, sejam por necessidade de auxílio financeiro, físico ou afetivo, afetam diretamente a forma com que cada uma vivencia suas experiências sociais e familiares, que pode criar laços de dependência (ALBERTE; RUSCALLEDA; GUARIENTO, 2015; CAUDURO, A.; BÓS; CAUDURO, M., 2013).

Por outro lado, melhores condições socioeconômicas podem influenciar positivamente no estado psicológico, nos domínios físicos e nas relações 
sociais, colaborando para que as idosas residam sozinhas. A dependência pode ser resultante do ambiente físico em que o idoso está inserido, do grau de proporção de segurança dos locais, que são suscetíveis à depressão e ao isolamento social, sendo que a última, tem conexão com o enfraquecimento da saúde física e mental (MONDENEZE et al., 2013; CAUDURO; GONÇALVES; CAUDURO, 2013; DAWALIBI; GOULART; PREARO, 2014; VITORINO; PASKULIN; VIANNA, 2013).

Constatou-se na pesquisa que quase a totalidade das idosas não recebiam ajuda financeira e mantinham os familiares com a sua renda principal, que era a aposentadoria. O que corrobora com estudo realizado em Recife (PE) por Cartaxo et al. (2012), no qual 75,5\% relataram ser pensionistas e/ou aposentadas. Em relação à renda familiar mensal, 59,5\% referiram receber até dois salários mínimos, além da renda proveniente da pensão e/ou aposentadoria, bem como os familiares que moram e trabalham com as idosas.

É importante destacar que as dificuldades financeiras impossibilitam a autonomia da idosa e podem desencadear problemas psíquicos, devido a percepção de desigualdade social, e à sentimentos de inutilidade e baixa autoestima. Isso pode afetar a saúde, uma vez que, um maior nível de renda permite desfrutar de melhor status de saúde, pela melhor aquisição de bens e serviços, condições de educação e de moradia. Em contrapartida, o acesso ao serviço público, também propicia maior nível de saúde principalmente aos desfavorecidos economicamente.

O estudo mostrou que a maioria das participantes possuía casa própria e morava com mais de três familiares. O que também foi visto em estudo feito em São Paulo, por Santos e Cunha (2014), apontando para a tendência das idosas residirem com muitos familiares atualmente. Assim como em Recife (PE), onde somente $2,7 \%$ das idosas residiam em casa alugada, demonstrando que apesar da baixa renda, ainda possuíam um imóvel (CARTAXO et al., 2012).

As pesquisas têm demonstrado também que os idosos com aposentadoria moram em aglomerado de pessoas, devido ao empobrecimento familiar, que faz com que os cuidadores tendam a morar com eles, já que dependem do recurso da aposentadoria e/ou pensão para sobreviverem (CAUDURO; GONÇALVES; CAUDURO, 2013; POLARO et al., 2013). No município de Guarapuava-PR, por exemplo, 54,3\% das idosas moravam na residência acompanhada por algum familiar, como filha/o, cônjuge e genro/nora (MACHADO; VIEIRA, 2015).

Na região de Guanambi (BA), as idosas possuíam, em sua maioria, o ensino fundamental, como também em outras regiões do país, como o estado de São Paulo, que 43,4\% completaram o ensino médio (SANTOS; CUNHA, 
2014). Em outros achados, $51 \%$ das idosas eram analfabetas e $49,0 \%$ possuíam o ensino fundamental incompleto (DAWALIBI; GOULART; PREARO, 2014).

Sabe-se que a escolaridade pode contribuir para um envelhecimento mais autônomo, pois viabiliza melhores oportunidades de emprego e contribui para uma melhor administração da renda, minimizando o impacto da condição socioeconômica em relação a sua qualidade de vida. Apesar disso, a maioria das idosas do estudo possuía baixa escolaridade, o que evidencia o contexto histórico de acesso à educação desse público algumas décadas atrás. A escolaridade permite também uma maior compreensão sobre a promoção da saúde e prevenção de doenças, fortalecendo assim, sua autonomia.

Uma pesquisa realizada na Grã-Bretanha apontou que a mulher é mais propensa a viver sozinha do que o homem, pois 30\% das mulheres entre a faixa etária de 65 e 74 anos, viviam sozinhas, na medida em que $20 \%$ dos homens nessa mesma idade. Esse fenômeno ocorre normalmente porque os homens nessa mesma faixa etária, preferem se relacionar com um cônjuge mais jovem. Diferente das mulheres idosas que tendem a permanecerem viúvas ou sozinhas, após a morte do cônjuge (CAUDURO; GONÇALVES; CAUDURO, 2013; SOUZA et al., 2015).

Como nessa faixa etária a fonte de renda é predominantemente a aposentadoria, muitas idosas vivem em pobreza extrema, muitas vezes, para garantir suas condições de sobrevivência e não reduzir seu padrão de vida, são obrigados a permanecer no trabalho (SILVA et al., 2013; SANTOS; CUNHA, 2014). No estudo realizado em Minas Gerais, constatou que os idosos tinham dificuldade em custear suas despesas, visto que recebiam somente um salário mínimo, sendo a pobreza, uma característica marcante dessa população (SILVA et al., 2013).

Entende-se que a condição econômica, está relacionada ao menor acesso ao serviço de saúde e vulnerabilidade social, aos fatores que ocasionam o comprometimento funcional e a maior incidência de fatores de risco e suscetibilidade a problemas de saúde. Nesta percepção, a baixa renda significa perda da eficiência para cuidar de si próprio e na convivência social, uma vez que a falta de recursos para o autocuidado e aumenta consideravelmente a proporção de doenças crônicas não transmissíveis, como a dependência ou depressão (MACHADO; VIEIRA, 2015; AMARAL; MELO; OLIVEIRA, 2015).

Sob esse ponto de vista, um estudo realizado no Irã, que analisou os fatores relacionados à qualidade de vida da idosa, verificou que a infelicidade e a dificuldade de relacionamento social entre as idosas era decorrente do menor nível educacional, que evidencia uma maior prevalência de analfabetismo entre 
idosas, com aproximadamente 37,4\% entre mulheres e 32, 2\% entre homens (VITORINO; PASKULIN; VIANNA, 2013; SANTOS; CUNHA, 2014).

Os estudos têm demonstrado que as idosas que possuem uma renda familiar menor do que dois salários mínimos têm maiores chances de residirem sozinhas do que aquelas com renda maior que dois salários mínimos. Porém, em relação à renda individual, quanto maior for a renda, maior será a probabilidade de viver sozinha. Nos países desenvolvidos, o número de idosas que vivem com os filhos diminui consideravelmente com o passar da idade, assim, os países em desenvolvimento, mesmo com a longevidade, continuam possuindo uma elevada proporção de idosas que moram com os filhos (CAUDURO; GONÇALVES; CAUDURO, 2013; SANTOS; CUNHA, 2014).

De fato, há um maior número de idosas na pirâmide etária populacional, tanto de países em desenvolvimento, quanto em desenvolvidos, resultante do maior uso de bebidas alcoólicas e tabaco, menor assistência médica e exposição dos jovens homens à mortalidade decorrente de causas externas como trânsito e homicídio. Essa prevalência do sexo feminino é um fenômeno internacional, a exemplo de estudos em Alemanha, Canadá e, especialmente com o passar da idade (AMARAL; MELO; OLIVEIRA, 2015; OLIVEIRA; NOVAES, 2013; VITORINO; PASKULIN; VIANNA, 2013; SANTOS; CUNHA, 2014).

A história e o contexto social em que a mulher cresce e envelhece predispõe à maior exclusão da idosa da participação política e social. Nesse contexto, o casamento foi e ainda se caracteriza como um vínculo de segurança inerente às exigências sociais. Porém, observa-se em estudos o aumento progressivo de idosas sem cônjuge, a exemplo das solteiras, separada e viúvas, que diferente dos idosos, costuma permanecer sozinhas após a viuvez ou separação.

Viu-se que grande parte das idosas participantes da pesquisa não apresentava cônjuge. Em outro estudo, percebeu-se que a maioria das idosas eram solteiras, separada ou viúvas, ou seja 71,7\% não apresentam cônjuge (CARTAXO et al., 2012). Nota-se nestes estudos, uma prevalência no número de idosas sem parceiros, o que compromete o estado emocional e físicos, que pode desencadear o isolamento social e a depressão.

Levando-se em consideração a afirmação acima, a morte do companheiro de longos anos, significa uma perda importante para as mulheres idosas, que resulta comumente em um luto prolongado, ocasionando sentimento de tristeza, solidão e adoecimento. Em contrapartida, os idosos casados possuem um nível maior de expectativa de vida e de bem-estar do que os-não casados, devido a assistência e os cuidados que o cônjuge presta em situações de doença e nos enfraquecimentos físicos (SOUZA et al., 2015; FREITAS et al., 2012). 
Além do convívio social e familiar e das condições socioeconômicas, outros fatores são essenciais para envelhecer com independência e autonomia. As atividades físicas e o lazer, por exemplo, uma vez que promovem a socialização da idosa, tornando-a ativa, auxiliam na percepção de controle e diminuição de sintomas depressivos e proporciona um desenvolvimento da saúde mental e físico. Já a ausência ou limitação do lazer ocasiona o sedentarismo e compromete a capacidade funcional da idosa (CABRAL et al., 2013; KHOURY; SÁ-NEVES, 2014; DAWALIBI; GOULART; PREARO, 2014; VITORINO; PASKULIN; VIANNA, 2013).

Neste estudo, os fatores relacionados à qualidade de vida das idosas, foram a renda, a percepção da saúde, lazer e a condição de moradia. Corroborando com outros estudos, como um realizado em São Paulo, confirmando que $70,1 \%$ das idosas não tinha acesso às atividades de lazer, enquanto apenas $19,9 \%$ das idosas praticavam atividade física. Ou em Betim-MG, no qual 79,5\% dos idosos foram classificados como sedentários (SANTOS; CUNHA, 2014; OLIVEIRA; NOVAES, 2013).

Ressalta-se que as atividades de lazer são fundamentais para melhorar a qualidade de vida, pois influenciam positivamente na autoestima e na saúde física e mental da pessoa idosa, devido ao compartilhamento de atividades com outras pessoas, possuindo uma forte interferência sobre a socialização. É notório que há um progressivo aumento da população idosa no Brasil, principalmente do segmento feminino. Assim, considerando-se que, o processo de envelhecimento é resultado de uma diminuição de execução de práticas laborais, psíquicas e biológicas, logo, os idosos tornam-se mais suscetíveis a desenvolver alguma dependência, seja física ou mental, caso não seja favorecido o envelhecimento com qualidade de vida. Qualidade de vida esta, que está vinculada aos diversos fatores que aumentam a expectativa de vida, que estão relacionados às condições de melhoria da autonomia das idosas.

\section{Conclusão}

A partir do presente estudo foi notório que os fatores sociodemográficos, como: sexo, idade, escolaridade, renda, estada civil e cor, bem como condições de saúde: autopercepção da saúde e o estilo de vida: atividade física e lazer estiveram relacionados com a qualidade de vida em mulheres idosas de Guanambi (BA), os quais propiciam maior acessibilidade ao serviço de saúde, melhores hábitos de vida e condições físicas e mentais, atuando assim, na promoção da saúde e prevenção de doença. 
O estudo destacou as questões que interferem diretamente no envelhecimento com qualidade de vida da mulher, como a escolaridade, que é um importante indicador para a adoção de comportamentos saudáveis, que promove a saúde e a melhoria das condições de vida; e o lazer, que permite a interação social nas relações interpessoais, assumindo compromissos de modo enriquecedor e criativo, com inserção no meio sociocultural.

É perceptível a necessidade de atenção à pessoa idosa e é imprescindível a implementação de ações que visem melhorar a qualidade de vida deste público tão crescente em todo o mundo. Ressaltando a importância da promoção de formas de envelhecimento ativo na sociedade, que permanece com a percepção da idosa sedentária, isolada socialmente e dependente. Sugere-se que outros estudos abordem as diferenças significativas entre a inserção do idoso na sociedade atual, correlacionando o gênero e o melhor acesso a equipamentos de saúde, e ainda a maiores oportunidades de vivenciar o envelhecimento ativo e com qualidade de vida.

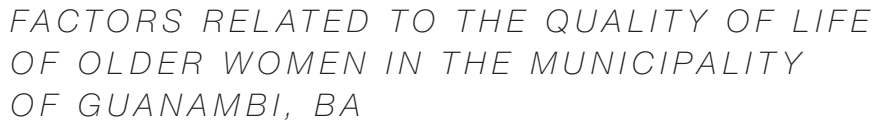

Quality of life is a set of subjective and objective factors, which should be evaluated, especially in aging, to understand the life and health needs of older adults and propose strategies to improve the quality of life of each individual. Objectives: to analyze the factors related to the quality of life of older women in the city of Guanambi, BA, in the year 2016. Materials and Methods: a quantitative cross-sectional study with a descriptive character, carried out with 550 older women. The instruments used in the data collection were a semi-structured form of situational diagnosis and the World Health Organization Quality of Life (WHOQOL-Bref) to measure the quality of life of this population segment. The analysis was performed using univariate statistics, with chi-square $\chi^{2}$ calculations, and simple logistic regression. Results: The older adults were mostly illiterate, retired, without spouses, black, living in multigenerational households and $68.2 \%$ with a positive perception about their quality of life, even living in adverse situations. The factors that were related to quality of life were sociodemographic, health conditions and lifestyle, and these factors negatively contribute to greater incapacity, vulnerability and dependence of the older 
adults. Conclusion: The well-being of the older adults is affected economic dimensions, with this, the research signaled the need to implement actions aimed at health care, recreation and socialization activities, which allow autonomy and independence, to improve the quality of life.

keywords

Risk Factors. Population Ageing. Women's Health.

\author{
referências
}

ALBERTE, Josiane de Souza Pinto; RUSCALLEDA, Regina Maria Innocencio: GUARIENTO, Maria Elena. Qualidade de vida e variáveis associadas ao envelhecimento patológico. Revista da Sociedade Brasileira de Clínica Médica, São Paulo, v. 13, n. 1, p. 32-39, jan./mar. 2015.

ALVES, Ana Laura A. et al. Qualidade de vida de vítimas de trauma seis meses após a alta hospitalar. Revista de Saúde Pública, São Paulo, v. 43, n. 1, p. 154-160, fev. 2009.

AMARAL, Tânia Maria de Resende; MELO, Elza Machado de; OLIVEIRA, Graziella Lage. Comparação do perfil de idosos ativos e não ativos do Programa Bolsa Família. Revista Brasileira de Geriatria e Gerontologia, Rio de Janeiro, v. 18, n. 2, p. 351-360, 2015.

CABRAL, Rômulo W. Lima et al. Fatores sociais e melhoria da qualidade de vida dos idosos: revisão sistemática. Revista de Enfermagem UFPE online, Recife, v. 7, n. 5, p. 1434-1442, maio 2013.

CAMPOS, Ana Cristina Viana; FERREIRA, Efigênia Ferreira; VARGAS, Andréa Maria Duarte. Determinantes do envelhecimento ativo segundo a qualidade de vida e gênero. Ciência \& Saúde Coletiva, Rio de Janeiro, v. 20, n. 7, p. 2221-2237, jul. 2015.

CAMPOS, Ana Cristina Viana; GONÇALVES, Lucia Hisako Takase. Perfil demográfico do envelhecimento nos municípios do estado do Pará, Brasil. Revista Brasileira de Enfermagem, Brasília, DF, v. 71, supl. 1, p. 591-598, 2018.

CARTAXO, Hemília Gabrielly de Oliveira et al. Percepção de idosas sobre o envelhecimento com qualidade de vida: subsídio para intervenções públicas. Revista da Rede de Enfermagem do Nordeste, Fortaleza, v. 13, n. 1, p. 158-168, 2012.

CASTRO, Susane Fátima Ferreira de; BARROS JúNIOR, Francisco Oliveira. Atenção à Saúde do Idoso: o pensar e o fazer Políticas Públicas. Revista Kairós: Gerontologia, São Paulo, v. 19, n. 22, p. 271-294, 2016.

CAUDURO, Adroaldo; BÓS, Ângelo José Gonçalves; CAUDURO, Maria Heloisa Fialho. Fatores associados a morar sozinho e suas diferenças regionais em idosos residentes de Porto Alegre e Manaus. Estudos Interdisciplinares sobre o Envelhecimento, Porto Alegre, v. 18, n. 2, p. 349-365, 2013.

CHAIMOWICZ, Flávio. Saúde do idoso. 2. ed. Belo Horizonte: Nescon UFMG, 2013.

DAWALIBI, Nathaly Wehbe; GOULART, Rita Maria Monteiro; PREARO, Leandro Campi. Fatores relacionados à qualidade de vida de idosos em programas para a terceira idade. Ciência \& Saúde Coletiva, Rio de Janeiro, v. 19, n. 8, p. 3505-3512, ago. 2014 
FEHLBERG, Maria da Penha Almeida. Mulheres idosas e suas histórias de vidas sofridas: direito a adoecer com cidadania;? quem sabe um dia... Revista Kairós: Gerontologia, São Paulo, v. 16, n. 5, p. 269-292, 2013.

FREITAS, Clara Maria Silvestre Monteiro et al. Identidade do idoso: representações no discurso do corpo que envelhece. Estudos Interdisciplinares sobre o Envelhecimento, Porto Alegre, v. 17, n. 1, p. 19-35, 2012.

GlL, Antônio Carlos. Como elaborar projetos de pesquisa. 4. ed. São Paulo: Atlas, 2010. INSTITUTO BRASILEIRO DE GEOGRAFIA E ESTATÍSTICA (IBGE). Censo demográfico 2010. Rio de Janeiro: IBGE, 2010.

INSTITUTO BRASILEIRO DE GEOGRAFIA E ESTATISTTICA (IBGE). Estimativas da população residente para os municipios e para as unidades da federação brasileiros com data de referência em $1^{\circ}$ de julho de 2015. Rio de Janeiro: IBGE, 2015.

KHOURY, Hilma Tereza Tôrres; SÁ-NEVES, Ângela Carina. Percepção de controle e qualidade de vida: comparação entre idosos institucionalizados e não institucionalizados. Revista Brasileira de Geriatria e Gerontologia, Rio de Janeiro, v. 17, n. 3, p. 553-565, set. 2014

KÜCHEMANN, Berlindes Astrid. Envelhecimento populacional, cuidado e cidadania: velhos dilemas e novos desafios. Sociedade e Estado, Brasília, DF, v. 27, n. 1, p. 165-180, abr. 2012.

MACHADO, Adrielli; VIEIRA, Maria Cristina Umpiérrez. Impacto de fatores socioeconômicos na funcionalidade da pessoa idosa portadora de condições crônicas. Revista de Enfermagem da UFSM, Santa Maria, v. 5, n. 1, p. 81-91, jan./mar. 2015.

MONDENEZE, Dênis Marcelo et al. Perfil epidemiológico e socioeconômico de idosos ativos: qualidade de vida associada com renda, escolaridade e morbidades. Estudos Interdisciplinares sobre o Envelhecimento, Porto Alegre, v. 18, n. 2, p. 387-399, 2013.

OLIVEIRA, Mirna Poliana Furtado de; NOVAES, Maria Rita Carvalho Garbi. Perfil socioeconômico, epidemiológico e farmacoterapêutico de idosos institucionalizados de Brasília, Brasil. Ciência \& Saúde Coletiva, Rio de Janeiro, v. 18, n. 4, p. 1069-1078, abr. 2013.

POLARO, Sandra Helena Isse et al. Dinâmica da família no contexto dos cuidados a adultos na quarta idade. Revista Brasileira de Enfermagem, Brasília, DF, v. 66, n. 2 , p. 228-233, mar./abr. 2013

PRODANOV, Cleber Cristiano; FREITAS, Ernani Cesar de. Metodologia do trabalho científico: métodos e técnicas da pesquisa e do trabalho acadêmico. 2. ed. Novo Hamburgo: Feevale, 2013.

SANTOS, Gerson Souza; CUNHA, Isabel Cristina. Avaliação da qualidade de vida de mulheres idosas na comunidade. Revista de Enfermagem do Centro Oeste Mineiro, Divinópolis, v. 4, n. 2, p. 1135-1145, maio/ago. 2014.

SILVA, Malu Emanuelle et al. Perfil epidemiológico, sociodemográfico e clínico de idosos institucionalizados. Revista de Enfermagem do Centro Oeste Mineiro, Divinópolis, v. 3, n. 1, p. 569-576, jan./abr. 2013.

SOUZA, Mariane de et al. A vivência da sexualidade por idosas viúvas e suas percepções quanto à opinião dos familiares a respeito. Revista Saúde e Sociedade, São Paulo, v. 24, n. 3, p. 936-944, 2015.

STEDILE, Taline; MARTINI, Maria Ivone Grilo; SCHMIDT, Beatriz. Mulheres idosas e sua experiência após a viuvez. Pesquisas e Práticas Psicossociais, São João del-Rei, v. 12, n. 2, p. 327-343, 2017. 
VAGETTI, Gislaine Cristina et al. Condições de saúde e variáveis sociodemográficas associadas à qualidade de vida em idosas de um programa de atividade física de Curitiba, Paraná, Sul do Brasil. Cadernos de Saúde Pública, Rio de Janeiro, v. 29, n. 5, p. 955-969, maio 2013.

VIEIRA, Kay Francis Leal et al. Representações sociais da qualidade de vida na velhice. Revista Psicologia: Ciência e Profissão, Brasília, DF, v. 32, n. 3, p. 540-551, 2012.

VISENTIN, Angelita et al. Qualidade de vida de idosas hipertensas de uma instituição de longa permanência. Revista da Rede de Enfermagem do Nordeste, Fortaleza, v. 16, n. 2, p. 218-225, mar./abr. 2015.

VITORINO, Luciano Magalhães; PASKULIN, Lisiane Manganelli Girardi; VIANNA, Lucila Amaral Carneiro. Qualidade de vida de idosos da comunidade e de instituições de Ionga permanência: estudo comparativo. Revista Latino-Americana de Enfermagem, Ribeirão Preto, v. 21, número especial, p. 3-11, jan./fev. 2013.

WORLD HEALTH ORGANIZATION (WHO). The WHOQOL Group. World Health Organization quality of life assessment (WHOQOL): development and general psychometric properties. Social Science \& Medicine, v. 46, n. 12, p. 1569-1585, 1998.

Data de Submissão: 14/05/2018

Data de Aprovação: 12/06/2019 
